\title{
I-SLATE: designing a culturally relevant framework for authentic learning
}

\author{
John Loewen ${ }^{1 *}$, Kinshuk ${ }^{2}$, Jarkko Suhonen ${ }^{1}$ and Nian-Shing Chen ${ }^{3}$
}

\author{
* Correspondence: \\ loewenj700@gmail.com \\ ${ }^{1}$ University of Eastern Finland, \\ Joensuu, Finland \\ Full list of author information is \\ available at the end of the article
}

\begin{abstract}
Many Indigenous learners worldwide find the learning materials presented in current educational settings culturally irrelevant. A lack of relevant learning materials within formal education settings subscribing to Western Euro-centric curriculum standards is a key factor. Learning materials provided through modelling, practice, and apprenticeship, are desired. With the literature identifying the lack of a formal curriculum design process, a framework is presented for standardized creation of learning objects for Indigenous learners. To determine the efficacy of the framework, two case study examples and prototype tools were presented to Indigenous knowledge expert interviewees to identify strengths, weaknesses and additional benefits to the proposed framework. Incorporating learners in the design process from the beginning was highlighted as being a very positive approach. The benefits of this research include that it provides an interface, in the form of a prototype, to allow educators to create relevant and authentic learning for Indigenous learners. Additional work is needed on simplifying the process of knowledge creation for educators and on ensuring that any knowledge used maintains cultural and contextual meaning to the communities in which it was generated.

Keywords: Indigenous knowledge, Educational technology, Framework, Learning objects, Fuzzy logic
\end{abstract}

\section{Introduction}

"I cannot read or write. I know nothing, but I know when a cyclone is coming from the north- east." - (Statement by a Bengali man, proclaimed in the documentary Savage Sea) (from Wane, 2008, p 183).

The educational systems currently encountered by many Indigenous learners are culturally irrelevant leading to a distinct lack of enthusiasm towards learning for many of these learners worldwide (Battiste, 2002). For the Indigenous learner, the bulk of learning materials provided by mainstream educational channels are delivered in a conventional, colonial, standardized way, often referred to as "Eurocentric" theory (Battiste, 2002). For example, in Canada there is a long history of colonization in many Indigenous communities resulting in imposed education systems often in the form of residential or boarding schools that resulted in a litany of abuses, and now intergenerational trauma. The result of this is that many Indigenous people identify formal education as a negative process, as a deliberate way to marginalize their languages, cultures, and distinct knowledge

(c) The Author(s). 2017 Open Access This article is distributed under the terms of the Creative Commons Attribution 4.0 International License (http://creativecommons.org/licenses/by/4.0/), which permits unrestricted use, distribution, and reproduction in any medium, provided you give appropriate credit to the original author(s) and the source, provide a link to the Creative Commons license, and indicate if changes were made. 
(Germain \& Dyck, 2011). Any educational initiative within Indigenous communities must take Indigenous knowledge into account when formulating and/or designing curriculum for learning. To most, Western knowledge and Indigenous knowledge are two very different knowledge systems but treating these as mutually exclusive, or as black and white, does not provide a useful way forward (Nakata, 2007). There is, in short, no single Indigenous knowledge 'theory' that might be analogous with Western knowledge and thought. Bridging these two seemingly monolithic knowledge structures is indeed a difficult task. Recent advances in software tools to analyze qualitative data for use in Indigenous knowledge research, for example, in agent-based, and artificial intelligence (AI) software that provides reasoning capabilities, suggest some possible ways forward (Sillitoe \& Marzano, 2009). Combining this with ways in which Information and Communications Technology (ICT) has been shown to be an effective tool for Indigenous learners (Donovan, 2007) is a key to creating learning opportunities for Indigenous learners that are both pedagogically and culturally relevant. ICT should benefit societies by allowing technological development without undermining local cultures or traditions and by supporting local identity rather than undermining it (Tedre et al., 2006). Therefore, understanding local culture and knowledge is a key component and any ICT system that is developed to support learning must be relevant to local needs, which need to be defined by local people and communities.

Knowing that Indigenous learners are not being adequately served in mainstream education, what is missing from the research is the concretization of a standardized methodology or a framework (particularly in the area of geographical and cultural context) for the storage and retrieval of Indigenous knowledge (Hartnell-Young \& Vetere, 2008). The purpose of this study is to propose a framework that leverages ICT to provide authentic and relevant learning opportunities for Indigenous learners. We present an approach that recognizes that a standardized framework approach to Indigenous learning may not work in all environments (Sillitoe \& Marzano, 2009), and that any approach must be flexible to gain the best fit between students, teaching and learning Nakata (2007). We propose an ICT-inclusive framework which may be leveraged to develop culturally relevant and authentic learning materials for Indigenous learners. Secondly, using the framework, we designed prototype learning tools and presented case study examples to Indigenous knowledge and curriculum experts in the field then performed one-on-one interviews using open-ended questioning techniques to gather data. From this data, qualitative data-analysis techniques were used to analyze the data to determine the efficacy of our approach.

\section{Review of related literature}

This literature review will first present the overlying concepts of the study to provide context and then delve into the work related to combining these overlying concepts towards applying the developed framework in an educational context.

\section{Indigenous knowledge and learners}

Indigenous knowledge (often referred to as traditional knowledge, local knowledge, traditional ecological knowledge, etc.) is a way of life, not an occupation or interest. Indigenous knowledge also resists specific definitions, as it is a fluid process, more than a defined thing and varies from community to community. Battiste (2002) describes Indigenous knowledge and thought as being experientially grounded, meaning that it is 
often oral and symbolic and is transmitted through the structure of Indigenous languages and passed on to the next generation through modelling, practice, and animation, rather than through the written word, and that it comprises the complex set of technologies developed and sustained by Indigenous civilizations. Many members of Indigenous communities live in rural areas and are raised in what would be considered an "unconventional" way by colonial Western standards, for example through apprenticeship, ceremonies, and practice in their environment (Daes, 1994) rather than in a classroom or laboratory (Kawagley and Barnhardt, 1999). Frustration, alienation, and failure increase when schooling imposes criteria oriented to success in Western society, without regard to Indigenous peoples cultural and community traditions (Wotherspoon \& Schissel 1998). Furthermore, these cultural and community traditions, often oral in nature, are disappearing with the extinction of the languages in which they are deeply associated, adding a component of urgency to the preservation of this valuable knowledge. With urgency mind, international agencies, such as the United Nations Development Programme (UNDP) promote ICT as a way in which to preserve this knowledge, and to facilitate access to education for remote populations (Clothey, 2015).

\section{Indigenous knowledge and ICT}

Sen (2005) identified ICT as a significant tool for the capture of Indigenous knowledge to facilitate both its preservation and access beyond person-to-person communication, for example, to document the traditional benefits and usage of medicinal plants. Some studies have suggested that Indigenous peoples are utilizing ICT to focus on the development of online communities and interfaces to tell stories and construct representations of self (i.e., Iseke-Barnes \& Danard, 2007; McLoughlin, 1999) (From Bang, Marin, Faber \& Suzukovich et al., 2013). Robbins (2006) demonstrates the importance of using ICT for contextual cultural learning in the South Pacific with 12 different indigenous groups (that are part of the University of the South Pacific). These educational tools include a virtual peer, wiki, self-test, digital scrapbook, and three-tier file structure. The concept of contextualization is discussed, which refers to the process of designing educational multimedia so that teachers and students can provide cultural context themselves. Learners and teachers create context in an ad-hoc way (for example, via a wiki), which requires their participation to work effectively. There are concerns around the preservation of Indigenous knowledge using ICT as often the contributors may not be aware of the cultural sensitivities related to the knowledge. Once the knowledge gathering process has commenced, it is then important to address the method or methods in which this Indigenous knowledge is organized and stored for the purpose of dissemination and retrieval within a culturally-relevant learning system (Sen, 2005). So what are some ways in which this knowledge may be used within such a learning system?

\section{Incorporating indigenous knowledge in to learning}

Berkes and Berkes (2009) identify fuzzy logic as a good fit for representing Indigenous knowledge in practice. Fuzzy logic provides a way in which to represent qualitative thought (for example observations of the environment used by Indigenous peoples) in a quantitative way (such as numerical data used in Western scientists in their observation of the environment). Grant and Berkes (2006) propose that Indigenous people have a knowledge system that can be described as an expert system, a branch of artificial 
intelligence (AI) that provides theories and methods for providing intelligent behaviour. Authors (2016) provide further insight in to how fuzzy logic may be used as a method in which to use fuzzy-logic to incorporate Indigenous knowledge in to learning.

Any system that does AI modeling and simulation or that takes pre-planning and an adaptable approach to ensure successful completion are labor and time intensive (Triantafyllakos et al., 2008). Lastly, when thinking of standardization as an approach for reusability, Sillitoe and Marzano (2009) observe that standard approaches may be problematic due to the diversity, internal variations, and constant revisions of knowledge and knowledge traditions world-wide, making generalizations potentially dangerous. Any approach towards formalization and standardization must be carefully considered. Further to this, the proper protocol of gaining the trust of an Indigenous community for the purpose of engagement in ICT initiatives can be a lengthy one (Srinivasan, 2007), sometimes taking years. This may include observations of community practice, attending meeting and functions within the community, and meeting with community members, particularly with Elders, to build bonds of connection and trust.

\section{Research gaps}

Hartnell-Young and Vetere (2008) identify that creating narratives that intertwine traditional literacies (for example, oral traditions) with new literacies (for example, storytelling via a mobile phone), has the potential for crossing boundaries between school and social contexts, which potentially allows students to include their uniquely cultural experiences to the curriculum, and conversely to take the knowledge from the curriculum to a broader social arena. The authors identify that to ensure a smoother integration of Indigenous knowledge in to formalized Western-based curriculum, pedagogical methods and best practices for applying them have to be identified. Pumpa et al. (2006) created a proof-of-concept prototype for archiving Indigenous knowledge in a digital landscape. As future work, the authors identified that the collaborative techniques used to create this content require more formalized research methodologies. Rathwell et al. (2015) identify that very little research has been performed in the area of incorporating contextual knowledge, such as Indigenous knowledge, into some kind of pedagogical framework. Further to this concept, Harrison and Greenfield (2011) identified that there was a need to define how teachers might incorporate "Indigenous knowledge" rather than "Indigenous perspectives" in their programs. The authors describe it as "to weave Aboriginal knowledge into the fabric" of the learning process in order to provide authentic learning opportunities for this style of learner. Rathwell et al. (2015) refer to this as "bridging" knowledge systems, for example, between Western and Indigenous knowledge, and identify this area of bridging Western knowledge and Indigenous knowledge as requiring more research, particularly in the area of multidisciplinary research that identify how knowledge systems are conceptualized.

Using these research gaps as a starting point, the following research questions emerged:

- What kind of theoretical and practical framework is appropriate for understanding the process of Indigenous knowledge transfer in the context of an Indigenous community?

- How may indigenous knowledge be seamlessly incorporated (inter-woven) in to current educational systems to provide relevant learning for Indigenous learners? 
By using a standardized framework as the pedagogical foundation for bridging knowledge systems, this study explores ways in which ICT may be leveraged to incorporate Indigenous knowledge in to Western curriculum to allow learning to occur in a relevant, authentic, and novel way. In particular, this study first presents a framework, the Indigenous Slate of Learning Authenticity Tools for Education (I-SLATE) framework, and determines the efficacy of the framework through the creation of a case study example and prototype tools that are presented to Indigenous knowledge and educational experts with the purpose of identifying to what extent they meet the study criteria.

\section{The I-SLATE framework}

ICT should benefit societies by allowing technological development without undermining local cultures or traditions and by supporting local identity rather than undermining it (Tedre et al., 2006). Therefore, understanding local culture and knowledge is a key component, and any ICT system that is developed must be relevant and reflective of local needs, which need to be defined by local people and communities. Working from the proposed framework identified by Authors (2016), the I-SLATE framework, shown in Fig. 1, attempts to bridge knowledge systems by standardizing a way in which to generate Indigenous knowledge and to incorporate it in to non-Indigenous curriculum in an authentic and relevant way.

Each of the 5 main phases to the I-SLATE framework are defined as follows:

1. Trust Building - The process of engagement and building strong relationships of trust is paramount, and sufficient time must be allocated for allowing this to happen (Maher, 2013), to allow for formal channels to be followed and permission granted for requesting community knowledge.

2. Knowledge Building - Once this trust has been established, knowledge gathering processes and/or methods may be used to encourage knowledge holders to share

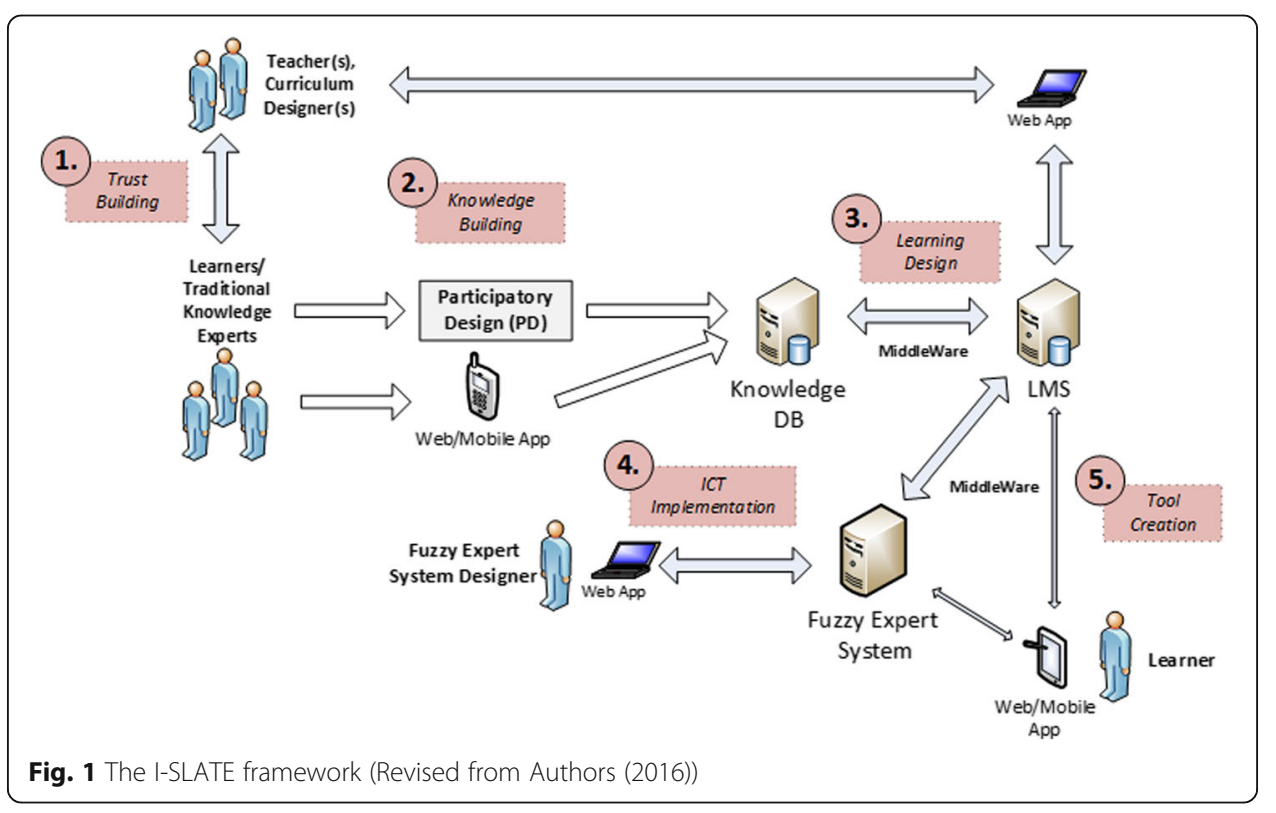


and/or acquire (from other community members) information on a particular branch of knowledge. As an example, participatory design (PD) has been shown to be a successful method of inquiry in this domain (Duveskog et al., 2009). Knowledge holders, knowledge sharers and community leaders play an important part in the knowledge generation process, which in order to acquire a depth of knowledge, is time-consuming and intensive.

3. Learning Design - Once the Indigenous knowledge has been gathered and entered in to the knowledge system, it may be used within relevant curriculum, for example, to create authentic learning objects that may conform to a standard format, such as IMS-LD, IEEE LOM or SCORM, allowing for reusability.

4. ICT Implementation -The creation of a smart learning environment (SLE). A fuzzy expert system is created using an interactive tool which is an interface (web-based in nature) that allows for the creation of fuzzy-logic rule-sets according to the communities knowledge base about a particular learning topic. The knowledge is used to create heuristic rule-sets that form the basis of a fuzzy expert system, defined previously by Authors (2016) providing the foundation for the creation of a simulation tool that may be used as an educational tool to complement other learning methods. The ICT implementation phase has the following components:

- Inputs - referred to as attributes, they are assigned an associated weight depending on their expected effect on the output, as determined through collaboration with community and community experts.

- Ruleset Creation - assigned by the fuzzy-logic and/or curriculum and combined together in the form of rule-sets. Rule-sets are combined using IF... THEN... ELSE logic to produce an output value, in the form of a linguistic value. This process forms the foundation of the fuzzy logic engine.

- Outputs - in the form of a linguistic value (for example, poor, average, good). The linguistic values may then be associated with learning object(s) or module(s), for example, Euro-centric learning objects (already in the curriculum) or Indigenous learning objects that were created during the Learning Design phase. These learning objects may be incorporated into a learning tool, such as a visual simulation tool and presented to the learner, as described by Authors (2016).

5. Tool Creation - A visual simulation tool is created to allow learners to provide inputs for the rulesets created in phase 4, allowing for simulations to be run and learning objects to be retrieved based on the output of the simulation. For example, visual tools that provide a sense of place have shown to be beneficial for Indigenous community members (Tripathi \& Bhattarya, 2004), therefore, a web and mobile map-based tool was created to allow learners to navigate through relevant landscapes (for example, traditional territories) and to provide inputs relevant to location or place.

Initially, the creation of the rule-sets will require an expert in fuzzy-logic expert systems. The framework designed is generic in nature, allowing for the knowledge to be interchangeable regardless of the rulesets. It is important to note that these 5 phases are fluid and may be performed iteratively over time to best fit the learning environment.

To provide proof of concept, to link the I-SLATE framework to authentic learning opportunities, and to represent the differing contexts in it may be used, two case study 
examples and prototype tools are presented. To demonstrate the viability and flexibility of the framework, the case studies identify the knowledge gathering process followed to determine the inputs, attribute values and outputs, and how these components are incorporated in to the I-SLATE framework to allow for the creation of authentic and relevant learning objects.

\section{CASE 1 - Salmon and the carrier people}

\section{Trust building and knowledge building}

The purpose of this case study is to simulate the effects of salmon harvest health on the health and well-being of a community. As the trust building phase is one that is built over a long period of time, (Maher, 2013), for the purposes of this case study, we are using knowledge that has been acquired from those who were able to build sufficient trust with a community over a period of many years. To provide historical perspective, according to Morice (1889, p 128) when speaking of the Nakaztli people of the Pacific Northwest;

"salmon is to the Carrier what wheat is to the white man - give them a large run of salmon, and abundance with its logical associates, rejoicing, feasting and dancing reign in the camp; cut off the supply, and there will be famine and desolation, silence in the village and melancholy in all hearts."

A bad harvest of salmon would directly affect the economic well-being of an individual, family and community. However, this was not the case for the Sekani people, whose traditional lands are roughly to the northeast of the Nakaztli. Morice (1889, p 128) observed that "the Sekanis disdain fish of any kind and regard fishing as a degrading occupation unworthy of a hunter". So in a historical context, "abundance" or "wealth" was (and in many cases, still is) defined much differently from community to community. Therefore, any learning system must take in to account the major local contextual differences between communities.

\section{Learning design}

The learning design phase incorporates the knowledge gathered into predefined curriculum (for example, as learning objects) or as standalone curriculum. For example, defining what "abundance" and "wealth" means to an individual community. Once these learning objects have been created, they may be incorporated in to the ICT Implementation phase as learning objects that are associated with an output linguistic value. To demonstrate a way in which learning design may be standardized within the i-SLATE framework, we created a web-based learning object tool that allows for the creation of standardized learning objects, as shown in Fig. 2.

These learning objects may be created from within an LMS and may conform to a well known standard, for example IMS-LD (in our case) or SCORM.

\section{ICT implementation}

Fuzzy logic rulesets may be created first by determining the attributes associated with the health of a seasonal salmon harvest, as determined during the knowledge building phase. A web-based ruleset generator has been created and may be accessed in a 
Welcome John. You are an instructor!

Learning Simulator - Learning Objects

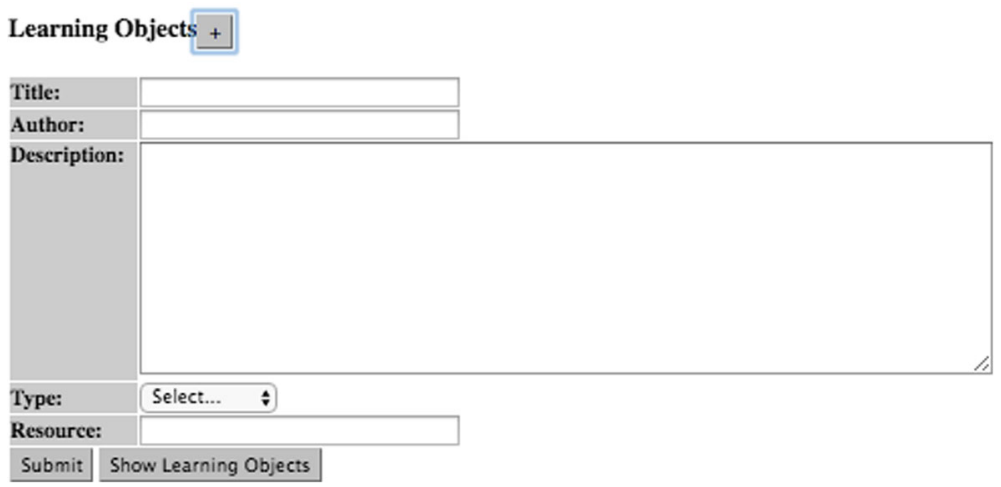

Fig. 2 LMS learning object creation (conforming to IMS-LD standard)

standardized way from within an LMS. Figure 3 shows an example of the ruleset generator accessed from the Moodle LMS using IMS Learning Tools Interoperability (LTI).

IMS-LTI provides a standardized way in which to integrate rich learning applications (for example, from remote locations) with platforms such as an LMS.

Inputs

All of the attributes that affect the health of a seasonal salmon harvest. An example set of attributes is provided in Table 1.

The attributes, linguistic values and weight applied in Table 1 have been arbitrarily created in consultation with a practitioner in the field. In practice, these values would be determined through participatory design techniques with community members.

\section{Ruleset creation}

An example sample ruleset, using the attributes from Table 1 would be:

- IF catch-per-unit-effort low THEN harvest-health good

- IF catch-per-unit-effort high THEN harvest-health poor

- IF catch-per-unit-effort normal THEN harvest-health moderate

\section{Financial Literacy Simulator}

You are an instructor!

Your user ID: 3

Rules +

IF weather $\quad \hat{\text { d dry }} \quad \mathbf{v}$ THEN harvest_health $\quad \hat{\text { poor }} \quad \hat{\mathbf{v}}$ Submit Rule

Refresh Rules

IF habitat-degredation IS low THEN harvest health IS low

IF by-catch IS moderate THEN harvest_health IS moderate

IF catch-per-unit-effort IS low THEN harvest health IS poor

IF harvest-mortality IS moderate THEN harvest health IS moderate

Fig. 3 Fuzzy logic aggregation example 
Table 1 Attributes for determining harvest health

\begin{tabular}{llll}
\hline Attribute (linguistic variable) & Linguistic values & Weight & Notes \\
\hline Habitat degradation & high, normal, low & 0.4 & $\begin{array}{l}\text { This affects ability to get up river and to } \\
\text { find spawning beds }\end{array}$ \\
Harvest mortality & high, moderate, low & 0.3 & Fish caught before they get to the community \\
Catch-per-unit-effort & high, normal, low & 0.5 & How easy are the fish to catch? \\
Weather & dry, seasonal, wet & 0.4 & $\begin{array}{l}\text { Affects the ability to start up river, also affects } \\
\end{array}$ \\
\end{tabular}

From this ruleset, a fuzzy rule base may be created that acts as the inference engine which steps through the heuristic rule sets one by one. The rule sets may be aggregated using ELSE IF logic. Multiple rulesets may then be aggregated to provide further reasoning, as shown in Fig. 4.

\section{Outputs}

The final step of the process is the "de-fuzzification" process. In this case study, 3 different rulesets are aggregated to determine the output value of harvest-health. The resulting numerical value is "de-fuzzified" to give it linguistic meaning, and then is used to retrieve the appropriate learning object(s) from the LMS database. These are the learning objects that were created during the Learning Design phase, and are shown in Fig. 4 as "LO Data".

\section{Tool creation}

The final phase of the framework is to create a learning environment that is culturally relevant. Visual tools that provide a sense of place have shown to be beneficial for Indigenous community members (Tripathi \& Bhattarya, 2004). A map-based tool

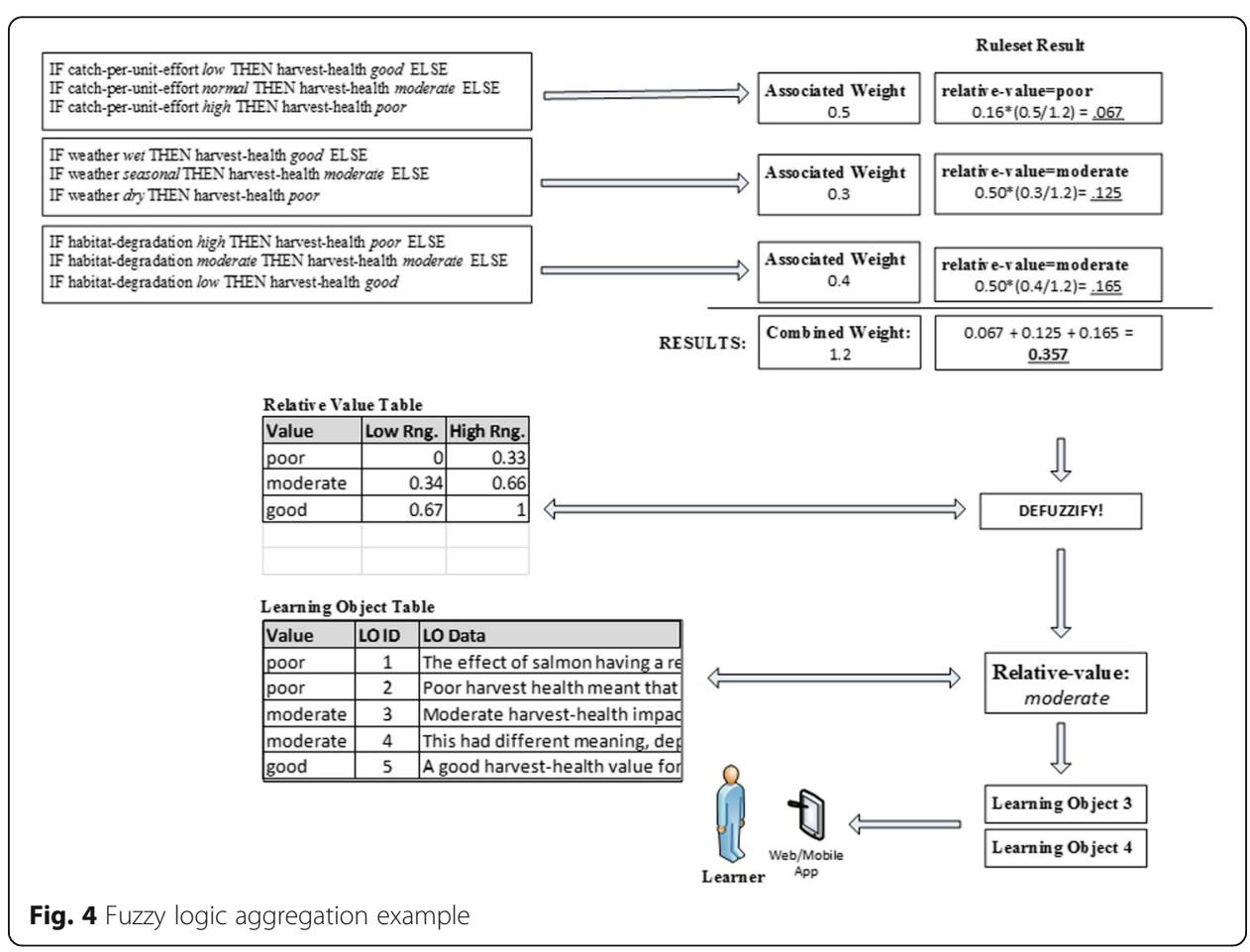


(in prototype form), shown in Fig. 5, has been created to allow learners to interact with their environment by providing simulations based on the fuzzy logic rulesets.

A simulation is then initiated with the output value (in this simple case created as poor, moderate, or good) linking to associated learning objects.

\section{CASE 2 - financial literacy - traditional vs. contemporary}

\section{Trust building and knowledge building}

Current methods of teaching financial literacy to communities lack cultural context and relevancy. Financial literacy from a Eurocentric perspective consists of a vocabulary that is not familiar, nor relevant to many Indigenous communities To a place-based holistic thinking community, the concept of "wealth" as it relates to financial literacy is dependent on the resources available to the community. There are many questions that need to be asked of a community before cultural context may be determined, for example, "what is/ was wealth?", "where and how were articles traded?", and "what is/was the value of articles of trade?" For example, terms such as 'economy' and 'budget' have origins in Western Eurocentric thinking. This case study simulates traditional trading mechanisms allowing the learner to acquire financial literacy through inclusive experiential learning. Data and knowledge in written form are scarce for pre-European contact trading, and because of this, data from currently accessible written archives are used for the purpose of this case study. In the 1800s there was extensive trading Between the Carrier People and the Hudson Bay Company (HBC) at Fort St. James, located in British Columbia, Canada. From the written logs kept, we can get an idea of the types, numbers and valuation of goods traded during certain years in this region. For example, from Morton (1988) we have a detailed record of the 1822 trading season at Fort Saint James, between the HBC and the Carrier People. A partial listing is shown in Table 2.

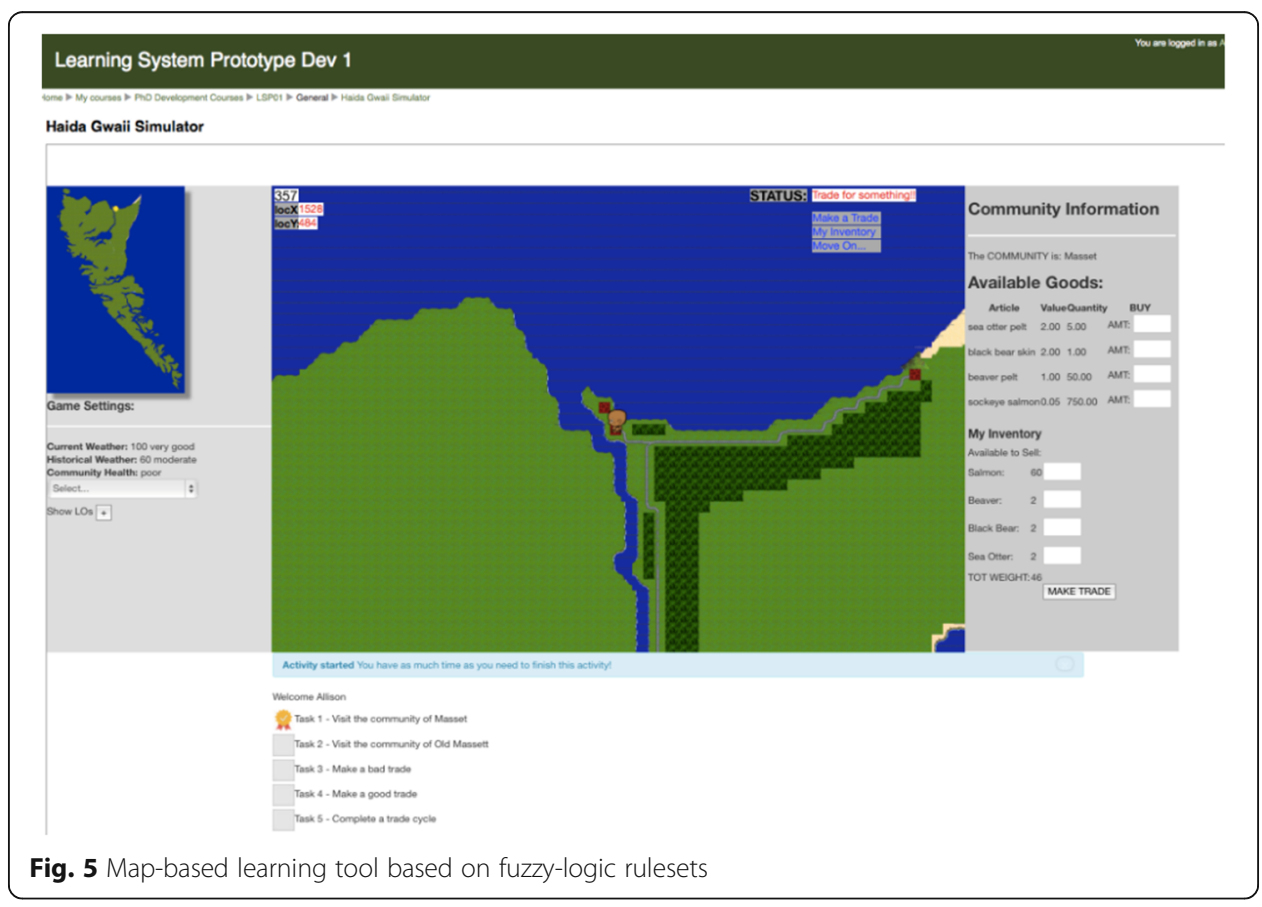


Table 2 Valuation of goods traded for at Ft. St. James, 1822, partial list (Source: Morton, 1988)

\begin{tabular}{lll}
\hline Article & Number of skins & Valuation \\
\hline Large Fine Beaver & 751 & 751 \\
Small Fine Beaver & 270 & 135 \\
Minks & 29 & 9.66 \\
Muskrats & 2015 & 201.5 \\
Large Blk Bear Skins & 32 & 64 \\
Large Grisly (sic) Bear Skins & 5 & 10 \\
\hline
\end{tabular}

During this period, the unit of currency from which all other valuations were determined was "one made beaver pelt" (Morton, 1988). Referring to Table 2 it can be determined that one large black bear pelt was worth 2 beaver pelts, 10 muskrat pelts worth 1 beaver pelt, etc. Fluctuations in value were determined by supply and demand, for example, in 1851, with the first major documented failure of the Fraser River sockeye run, sockeye salmon, an essential part of sustenance and economy for the Carrier, the value of sockeye salmon as a trade item increased from 90 salmon per beaver pelt to 50 salmon per beaver pelt, effectively almost doubling the "price" of salmon.

\section{Learning design}

The learning design phase incorporates the knowledge gathered into predefined curriculum that has been created from a Euro-centric perspective. Learning objects may be created from the knowledge gathered. For example, in our case, learning objects are created based on the historical relative value of certain objects during periods of trade between communities and the HBC. Once these learning objects have been created, they may be incorporated in to the ICT Implementation phase as learning objects that are associated with an output linguistic value, for example, relative-value.

\section{ICT implementation}

Fuzzy logic rulesets may be created first by determining the attributes associated with wealth of a community, as determined during the knowledge building phase. With the knowledge acquired, objects and their associated values are known.

\section{Inputs}

Inputs for the wealth of a community consist of knowledge related to traditional mechanisms of trading, specifically what, how, and when materials were traded. These factors are assigned weights according to their importance. A preliminary example set of attributes is provided in Table 3.

The attributes, linguistic values and weight applied in Table 1 have been created using knowledge obtained from the available literature. In practice, these values may be determined through participatory design techniques with community members. In order to obtain a more comprehensive set of knowledge, further questions must be asked of community members and experts, such as what other items were/are imported and exported between local communities, not just with the HBC. 
Table 3 Factors for determining the relative-value of an object

\begin{tabular}{llll}
\hline Attribute (linguistic variable) & Linguistic values & Weight & Notes \\
\hline Supply & high, moderate, low & 0.5 & $\begin{array}{l}\text { This affects the relative cost } \\
\text { of a particular artifact/object. } \\
\text { Demand }\end{array}$ \\
$\begin{array}{llll}\text { Quality } \\
\text { houte-difficulty }\end{array}$ & $\begin{array}{l}\text { This affects the relative cost } \\
\text { of a particular artifact/object } \\
\text { very low, low, moderate, low } \\
\text { high, very high }\end{array}$ & 0.3 & $\begin{array}{l}\text { Grading value related to quality. } \\
\text { An item of trade may have to } \\
\text { be transported from point A to } \\
\text { point B which can also be a } \\
\text { determinant of its relative value. } \\
\text { The more difficult the route, the } \\
\text { higher the relative value. }\end{array}$ \\
\hline
\end{tabular}

\section{Ruleset creation}

Created according to the relative value of articles of trade to each community. Someone knowledgeable in fuzzy-logic is needed to create the rulesets and then incorporate them into the mechanisms of trading, through such processes as one-to-one trading, the potlatch, and through fairs that were held on borders of certain territories (Morice \& Père, 1894). An example ruleset is shown in Fig. 3.

\section{Outputs}

The relative value of an object is calculated using fuzzy logic, and "de-fuzzified" according to the rules of trade determined through the participatory process and the resulting ruleset creation.

As with the first case study example, 3 different rulesets are aggregated to determine the output value of relative-value. The resulting numerical value is de-fuzzified to give it linguistic meaning, and then used to retrieve the appropriate learning object(s) from the learning object table.

\section{Learning design relevancy and authenticity}

The preceding two case studies highlight the value of leveraging ICT to allow for many different learning opportunities to be created in a short period of time by running simulations with varying inputs. For example, the traditional effects on supply and demand in a community may be simulated at different levels, resulting in different outputs from the simulation, providing multiple learning opportunities that may otherwise not be realized. As shown in Fig. 3, different outputs allow for the presentation of learning objects that are relevant to that specific output. To further examine the efficacy of this case study approach, a case study and prototype tools were presented to experts in the area of Indigenous knowledge, education, and IT fields, with the purpose of providing feedback on this approach.

\section{Research findings}

Qualitative research methods were used for this study, using questions designed in in order to elicit responses that would best reflect the experiences and knowledge of the participants in regards to the efficacy of our approach. This took the form of an interview and questionnaire process, performed by the authors, with the focus on deep qualitative data to provide more in-depth analysis of the concepts presented. This 
follows a phenomenological research approach, outlined by Creswell (2014) as consisting of three to ten participants with the purpose of determining the "essence of their experiences" (Creswell, 2014, p 14). One hour was allocated for each interview. Due to distance and cost restraints, the interview process was completed face-to-face or online. The format of each interview consisted of a 20-min overview and demonstration, followed by $40 \mathrm{~min}$ to complete 6 open-ended questions. Online interviews were provided with a 20-min video demonstration, to ensure that the participants were able to observe the visual components of the prototype tool. The questions were designed to initiate dialog with the intent of acquiring rich qualitative data for analysis. A complete list of the questions is shown in Appendix 1.

The research results in this section should not be seen as a rigorous evaluation of the proposed framework, but as reflections arising from the case studies presented as well as some qualitative insight to assist in the direction of further development of the I-SLATE framework.

\section{Participants}

Six participants (two males and four females) took part in the interview portion of this study. Three of the participants were Indigenous and three non-Indigenous. As this research process involves both Indigenous and non-Indigenous educators interfacing between two disparate knowledge systems, it was important to include both Indigenous and non-Indigenous participants. All participants chosen are highly-qualified in the educational and educational technology sectors, with their areas of expertise including:

- Lead, Community Engagement, Education and Evaluation for Aboriginal Health

- Director of Aboriginal Education and Community Connections (Tertiary Institution)

- Associate Professor, Educational Technology (Tertiary Institution)

- Faculty Development, Center of Excellence for Teaching and Learning (Tertiary Institution)

- Co-editor of English Practice Journal and Doctoral Student - Language and Literacy Education

\section{Strengths}

The strengths identified for this framework are firstly, that it includes the learners in the design process from the beginning, allowing for authentic learning, as the narrative that is created is the learners' narrative. Four of the six participants (two Indigenous and two non-Indigenous) identified this as a benefit of the system. As one participant stated;

"I think what you are doing is good work because you are building a level of engagement at the beginning rather than assessing the results at the end".

Further to this, the benefits of the system for the learner, teacher, and curriculum designer, the collaboration and co-production of knowledge from the beginning is seen as very important as it provides a social venue in which to share knowledge and ideas and that;

"my actions will benefit the collective; therefore we as a species will survive, if that collective is always connected to the environment". 
Another participant seconded this thought by stating that "you have to try and bring the social in to everything you do in Indigenous culture" Two of the six participants identified experiential learning techniques and real-life problem solving as relevant methodologies, as well as including the learners in the curriculum generation process. By providing a tool that presents a standardized interface through an LMS, which many students are now familiar with, the process of incorporating local content in to a course is simplified.

\section{Weaknesses}

Weaknesses identified by the participants include the lack of an underlying narrative or storyline, which if contextually relevant, adds an additional level of authenticity, and therefore interest, for the learners. It was identified by two participants (one Indigenous and one non-Indigenous) that further work was required on the fuzzy-logic component of the system, in the form of concretization of the examples. As one expert stated:

"where things bog down for me, as a non-IT person is in the 'fuzzy logic' system.

I don't understand it, it's more like a black box for me - that needs to be unwrapped and explained."

Where and how the knowledge is utilized is very important to clarify, along with how it is relevant to other community members learning from it. Further to this, there is a fear on how knowledge may be interpreted by an "outsider". One participant expressed this concern by saying:

"it would mean relying on the expert system programmer's interpretation s of what was relevant and/or meaningful to include".

In other words, ruleset creation would mean that the learners would be relying on the expert system designer's interpretation of the knowledge, which may be very different than the actual intent. Additionally, teachers will need to be educated on how to use the tools and how to create the appropriate learning objects for the subject matter. Lastly, there was concern that this tool might take away from actually connecting with the land and with community. As one participant stated:

"it's not outdoors. When you talk about trade/bartering, it involves being on the land, so how do you build that in?"

\section{Additional benefits}

Two of the six participants (one Indigenous and one non-Indigenous) identified that a "quest approach" with community-generated narrative would be beneficial. For example, a language quest that incorporated traditional language in to the learning process. As one expert explained;

"they (the learners) find words as treasure along their journey. The word has embedded meaning that also connects it to the land, and we are using this word because it also connects to a particular species, salmon for example". 
This approach is analogous to experience-based learning (EBL), with Andersen et al. (2000) stating that the most important goal being that it involves "learners' own appropriation of something that is to them personally significant or meaningful", with a primary focus on the nature of learners' personal engagement with phenomena. As Ruben (1999) observes, the many forms of EBL used in professional education include case study approaches and computer simulations among others, and involves a more participative and learner-centered approach, encompassing and it encompasses formal learning, informal learning, non-formal learning, and lifelong learning. McLoughlin (1999) adds to this notion saying that the adoption of the 'communities of practice model' for online delivery enables learners to have access to community knowledge, support structures and shared interests', which lends credence towards leveraging ICT as a solution. Digital storytelling was identified by multiple participants as a method towards engagement of community members, for example, creating movies about family and community connections. One participant stated:

\section{"I believe that using the technology as a tool to achieve genuine connection with community members is incredibly important for youth, particularly given the higher incidences of suicide in rural and remote Indigenous communities. Using technology to interview and create movies about their family and community connections is a way to achieve this."}

Three of the six participants identified that to provide cultural relevance, any tool must support students to engage directly with their environment and their community. And lastly, three of six participants (one Indigenous and two non-Indigenous) identified that an additional strength was the ability to use the knowledge obtained beyond just educational applications as the knowledge would be stored using ICT (for example in a relational database) making it accessible. One participant identified this knowledge as "being useful for other community needs, for example, treaty negotiations".

\section{Discussion}

The purpose of this research was to design and implement a framework where ICT may be leveraged to create authentic and relevant learning opportunities for Indigenous learners. The preceding case studies illustrated that the I-SLATE framework highlights two very important elements to the learning design process. Firstly, that authentic learning may occur through knowledge gathering, dissemination and learning object design. Secondly, that the authentic learning objects created through the development process may vary greatly from community to community; so much so that even communities within a very short geographic proximity may find that through the interface designed, authentic learning towards a particular subject or object may contrast greatly from one community to the next. So if authentic learning is desired, designing learning with community involvement at the forefront is an essential step. Using fuzzy logic provides a method for disseminating this knowledge in relevant and authentic ways for Indigenous learners. As Berkes and Berkes (2009) state, "Indigenous knowledge holders do not need fuzzy logic to understand holism; they already practice it." By simulating authentic learning opportunities, this research aims to demonstrate the value of using ICT to create a tool to allow for many different learning opportunities to be 
experienced in a short period of time by running simulations with varying inputs. Learning opportunities may be created on the fly by interfacing with relevant technologies, such as IMS-LD Level B, which allows for the inclusion of run-time IF-THEN-ELSE logic to access contextually and culturally relevant learning objects, called conditions, used for personalization and adaptation (Koper \& Burgos, 2005). Lastly, following the prescribed framework allows for iterative design, ensuring that previously created learning objects and tools may be built on (for example multiple ruleset aggregation), added to, and improved as the design process progresses. If a modular approach is taken and the proposed framework is followed, then once this initial stage is completed, libraries may be created and may be used and added to in future knowledge building exercises.

\section{Conclusions and future work}

Statistical data from Indigenous communities around the world show that due to very low success rates for Indigenous learners, the current methodologies for teaching Indigenous learners are not working. This is due to a lack of appropriate pedagogy and a lack of culturally relevant learning materials. Defining a way in which Indigenous knowledge, or local knowledge and ICT may be integrated to make sense of the world as approached by a holistic thinking culture (for example, Indigenous learners) is of great utility. This paper provides qualitative analysis of Indigenous knowledge and curriculum expert interviews with the purpose of providing feedback on the I-SLATE framework of Authors (2016). Two case studies and prototype learning tools were presented to expert participants to illustrate how the I-SLATE framework may be used in practice. Issues identified in previous literature include the initial setup of the framework and of the Indigenous knowledge data, which are both labor and time intensive and to this end, additional tools have been created to streamline this process. However, what has become clear in the analysis of this approach, particularly from Indigenous expert feedback, is that community trust-building and engagement must be at the fore-front of any attempt to apply this system in a specific context, and that any tool used must build on the principles of supporting learners to engage directly with their environment and their community. With these conditions being followed carefully, the benefits to the I-SLATE framework are, for example, that it may provide government decision makers a way in which to better understand how Indigenous knowledge is relevant to today's educational system; it may provide a system that nonIndigenous peoples can use to understand Indigenous knowledge systems; and, if a modular approach is taken and the proposed framework is followed, then once the knowledge building exercises are completed, libraries may be created, used and added to in future iterations.

Future work of interest may include research on approaches to imparting knowledge to the educators of the learners in the I-SLATE framework, as well as finding opportunities to apply the proposed framework in other educational environments where a large number of "big picture" variables make quantification of data very difficult, thereby allowing ICT, and specifically this framework, to do the "heavy lifting". From the feedback of Indigenous education experts, research on ways in which to use this system to support learners to engage with their environment and community, for example, through digital storytelling, would be valuable. The implications of our 
findings for future research, design theory, and practitioners trying to enact culturally relevant pedagogy is that it provides a framework that may be used to establish culturally-appropriate relationships to allow for the collection, storage, and retrieval of Indigenous knowledge, that through leveraging ICT, may be used to integrate Indigenous knowledge in to education in useful and novel ways, providing authentic and relevant learning opportunities for Indigenous learners.

\section{Appendix 1}

\section{Validation Interview Questions}

1. Please identify your please identify your professional roles and interests as well as any other affiliations and roles you would like to mention?

2. How have Indigenous approaches to knowledge shaped the ways you think about learning design? About how educational technology may be used in learning design?

3. Is there an alternative learning path that remote, rural Indigenous learners may follow that provides cultural relevance leading to higher levels of interest and engagement?

4. What do you see as the benefits/drawbacks of this system for the learner/teacher/ curriculum designer?

5. What do you see as the overall strengths/weaknesses of this approach to designing learning for Indigenous learners?

6. Are there other ways in which you could envision a system like this being used? Please elaborate if possible.

7. Is there anything else you would like to add or comment on?

Funding

There was no funding for this paper.

Authors' contributions

$J$ carried out all of the research and the studies and drafted the manuscript. All authors read and approved the final manuscript.

Ethics approval and consent to participate

Not applicable.

Consent for publication

Not applicable.

Competing interests

The authors declare that they have no competing interests.

\section{Publisher's Note}

Springer Nature remains neutral with regard to jurisdictional claims in published maps and institutional affiliations.

Author details

${ }^{1}$ University of Eastern Finland, Joensuu, Finland. ${ }^{2}$ University of North Texas, Denton, USA. ${ }^{3}$ National Sun Yat-sen

University, Kaohsiung, Taiwan.

Received: 14 November 2017 Accepted: 28 November 2017

Published online: 15 December 2017

References

L Andersen, D Boud, R Cohen, Experience-based learning. Understanding adult education and training 2, 225-239 (2000)

Authors (2016).

M Battiste, Indigenous Knowledge and Pedagogy in First Nations Education: A Literature Review with Recommendations (Apamuwek Institute, Ottawa, 2002) 
F Berkes, MK Berkes, Ecological complexity, fuzzy logic, and holism in indigenous knowledge. Futures 41(1), 6-12 (2009) RA Clothey, in Indigenous Education. ICT and indigenous education: Emerging challenges and potential solutions (Springer, Netherlands, 2015), pp. 63-75

JW Creswell, Research Design: Qualitative, Quantitative, and Mixed Methods Approaches (Sage publications, 2014)

EIA Daes, Equality of indigenous peoples under the Auspicies of the United Nations-draft declaration on the rights of indigenous peoples. Thomas L. Rev. 7, 493 (1994)

Donovan, M. (2007). Can information communication technological tools be used to suit Aboriginal learning pedagogies. Information technology and Indigenous people, 93-104.

M Duveskog, K Kemppainen, R Bednarik, E Sutinen, in Proceedings of the 8th International Conference on Interaction Design and Children. Designing a story-based platform for HIV and AIDS counseling with Tanzanian children (ACM, 2009), pp. 27-35

Germain, G. S., \& Dyck, L. E. (2011). Reforming First Nations Education: From Crisis to Hope. Standing Senate Committee on Aboriginal Peoples.

S Grant, F Berkes, Fisher knowledge as expert system: A case from the longline fishery of Grenada, the Eastern Caribbean. Fisheries Research 84(2), 162-170 (2007)

N Harrison, M Greenfield, Relationship to place: Positioning aboriginal knowledge and perspectives in classroom pedagogies. Critical Studies in Education 52(1), 65-76 (2011)

E Hartnell-Young, F Vetere, A means of personalising learning: Incorporating old and new literacies in the curriculum with mobile phones. Curric. J. 19(4), 283-292 (2008)

J Iseke-Barnes, D Danard, Indigenous knowledges and worldview: Representations and the Internet. Information technology and indigenous people, 27-29 (2007)

AO Kawagley, R Barnhardt, in Ecological Education in Action: On Weaving Education, Culture and the Environment, ed. by G A Smith, D R Williams. Education indigenous to place: Western science meets native reality (State University of New York Press, Albany, 1999), pp. 117-140

Koper, R., \& Burgos, D. (2005). Developing Advanced Units of Learning Using IMS Learning Design Level B.

M Maher, Making inclusive education happen: The impact of initial teacher education in remote aboriginal communities. Int. J. Incl. Educ. 17(8), 839-853 (2013)

C McLoughlin, Culturally responsive technology use: Developing an on-line community of learners. Br. J. Educ. Technol. 30(3), 231-243 (1999)

AG Morice, in Proceedings of the Canadian Institute. The Western Denes, their Manners and Customs (Copp, Clark, Toronto, 1889)

AG Morice, Notes archaeological, industrial and sociological on the Western Dénés: With an ethnographical sketch of the same (University of Alberta, Edmonton, Canadian Institute, 1893)

J Morton, Fort St. James 1806-1914: A Century of Fur Trade on Stuart Lake (Environment Canada, Canadian Parks Service, 1988)

M Nakata, The cultural interface. The Australian Journal of Indigenous Education 36(S1), 7-14 (2007)

M Pumpa, TG Wyeld, B Adkins, in Advanced Learning Technologies, 2006. Sixth International Conference on. Performing traditional knowledge using a game engine: Communicating and sharing Australian aboriginal knowledge practices (IEEE, 2006), pp. 810-814

K Rathwell, D Armitage, F Berkes, Bridging knowledge systems to enhance governance of environmental commons: A typology of settings. International Journal of the Commons 9(2) (2015)

C Robbins, Providing cultural context with educational multimedia in the South Pacific. Educational Technology \& Society 9(1), 202-212 (2006)

BD Ruben, Simulations, games, and experience-based learning: The quest for a new paradigm for teaching and learning. Simulation \& Gaming 30(4), 498-505 (1999)

KA Scott, K Clark, M Bang, A Marin, L Faber, ES Suzukovich, Repatriating Indigenous Technologies in an Urban Indian Community. Urban Education 48(5), 705-733 (2013)

B Sen, Indigenous knowledge for development: Bringing research and practice together. The International Information \& Library Review 37(4), 375-382 (2005)

P Sillitoe, M Marzano, Future of indigenous knowledge research in development. Futures 41(1), 13-23 (2009)

R Srinivasan, Indigenous, ethnic and cultural articulations of new media. International Journal of Cultural Studies 9(4), 497-518 (2016)

M Tedre, E Sutinen, E Kähkönen, P Kommers, Ethnocomputing: ICT in cultural and social context. Commun. ACM 49(1), $126-130(2006)$

GN Triantafyllakos, GE Palaigeorgiou, IA Tsoukalas, We! Design: A student-centred participatory methodology for the design of educational applications. Br. J. Educ. Technol. 39(1), 125-139 (2008)

Tripathi, N., \& Bhattarya, S. (2004). Integrating indigenous knowledge and GIS for participatory natural resource management: State of the practice. EJISDC: The Electronic Journal on Information Systems in Developing Countries, (17), 3.

NN Wane, Mapping the field of indigenous knowledges in anti-colonial discourse: A transformative journey in education. Race Ethnicity and Education 11(2), 183-197 (2008)

T Wotherspoon, B Schissel, Marginalization, Decolonization and Voice: Prospects for Aboriginal Education in Canada (Discussion Paper. Pan-Canadian Education Research Agenda. Council of Ministers of Education, Canada, 1998) 